\title{
Erratum to: Self-assembly and gelation of TX-100 in water
}

\author{
Rahul Ranjan ${ }^{1} \cdot$ Prasenjit Das $^{1} \cdot$ Kamla Rawat $^{2,3}$ • V. K. Aswal ${ }^{4}$ J. Kohlbrecher ${ }^{5}$. \\ H. B. Bohidar ${ }^{1,2}$
}

Published online: 4 May 2017

(C) Springer-Verlag Berlin Heidelberg 2017

\section{Erratum to: Colloid and Polymer Science}

\section{DOI: 10.1007/s00396-017-4078-9}

We have subsequently found an error in the name of one of the authors. His name has been wrongly printed as "Pasenjit Das". It should be read as "Prasenjit Das".

The online version of the original article can be found at http://dx.doi.org/ 10.1007/s00396-017-4078-9

Electronic supplementary material The online version of this article (doi:10.1007/s00396-017-4109-6) contains supplementary material,

which is available to authorized users.

\footnotetext{
Kamla Rawat

kamla.jnu@gmail.com

$\triangle$ H. B. Bohidar

bohi0700@mail.jnu.ac.in

1 School of Physical Sciences, Jawaharlal Nehru University, New Delhi 110067, India

2 Special Center for Nanosciences, Jawaharlal Nehru University, New Delhi 110067, India

3 Inter University Accelerator Centre, New Delhi 110067, India

4 Solid State Physics Division, Bhabha Atomic Research Centre, Mumbai 400085, India

5 Paul Scherrer Institut, 5232 Villigen, Switzerland
} 